\title{
VOLUME AND DURATION OF LOSSES IN FINITE BUFFER FLUID QUEUES
}

\author{
FABRICE GUILLEMIN, ${ }^{*}$ Orange Labs \\ BRUNO SERICOLA, ${ }^{* *}$ INRIA
}

\begin{abstract}
We study congestion periods in a finite fluid buffer when the net input rate depends upon a recurrent Markov process; congestion occurs when the buffer content is equal to the buffer capacity. Similarly to O'Reilly and Palmowski (2013), we consider the duration of congestion periods as well as the associated volume of lost information. While these quantities are characterized by their Laplace transforms in that paper, we presently derive their distributions in a typical stationary busy period of the buffer. Our goal is to compute the exact expression of the loss probability in the system, which is usually approximated by the probability that the occupancy of the infinite buffer is greater than the buffer capacity under consideration. Moreover, by using general results of the theory of Markovian arrival processes, we show that the duration of congestion and the volume of lost information have phase-type distributions.
\end{abstract}

Keywords: Fluid queue; Markov chain; congestion

2010 Mathematics Subject Classification: Primary 60J10

Secondary $80 \mathrm{M} 35$

\section{Introduction}

A key performance metric for characterizing statistical multiplexing in packet networks is the volume of information lost during congestion periods of the network. This is a global performance measure reflecting congestion phenomena occurring on each transmission link of the network when performing statistical multiplexing in order to optimize network utilization. As a matter of fact, several packet flows are statistically multiplexed on the same transmission link which is generally equipped with a buffer to store packets when the arrival rate is momentarily greater than the link transmission capacity. This is intended to prevent, as much as possible, the loss of packets from the transmission link. However, such a buffer must have a finite capacity and packets can be transmitted in bursts, causing transient overflow of transmission links. Such phenomena are becoming more and more frequent in the Internet since new versions of the transmission control protocol (TCP) are ever more aggressive for the network and the slowstart mechanism is sometimes disabled, leading to the transmission of bulk of data at very high rates.

In this paper we consider a single transmission link equipped with a buffer of finite capacity. To model the arrival of packets, a usual approximation consists of ignoring the discrete nature of packets and assuming that a continuous amount of fluid arrives at the buffer (fluid flow approximation). Let $\chi(t)$ denote the input rate at time $t$ and let $\Lambda(t)$ be the net input rate

Received 11 February 2014; revision received 18 July 2014.

* Postal address: Orange Labs, 2 Avenue Pierre Marzin, 22300 Lannion, France.

Email address: fabrice.guillemin@ orange.com

** Postal address: INRIA, Campus de Beaulieu, 35042 Rennes Cedex, France. Email address: bruno.sericola@inria.fr 
at time $t$ (i.e. the difference between the instantaneous arrival and server rates). The link is congested at time $t$ when $\Lambda(t)>0$ and the buffer capacity (denoted by $x$ ) is reached. Denoting by $X(t)$ the volume of information in the buffer at time $t$, the fraction of lost information is the long-run ratio

$$
\pi_{\text {loss }}=\lim _{t \rightarrow \infty} \frac{\int_{0}^{t} \Lambda(s) \mathbf{1}_{\{X(s)=x\}} \mathrm{d} s}{\int_{0}^{t} \chi(s) \mathrm{d} s},
$$

where $\mathbf{1}_{A}$ denotes the characteristic function of the set $A$. (Note that we can have $X(t)=x$ only if $\Lambda_{t} \geq 0$.) When the process $(\Lambda(t))$ is stationary and ergodic, the above equation can be written as

$$
\pi_{\text {loss }}=\frac{\mathbb{E}\left(\Lambda \mathbf{1}_{\{X=x\}}\right)}{\mathbb{E}(\chi)},
$$

where $\chi, \Lambda$, and $X$ denote the input rate, the net input rate, and the buffer occupancy in the stationary regime, respectively.

The major difficulty for estimating the quantity $\pi_{\text {loss }}$ is the computation of the joint probability distribution of the couple $(\Lambda, X)$. One classical approach consists of approximating the quantity $\pi_{\text {loss }}$ by the buffer overshoot probability $\mathbb{P}\left\{X_{\infty}>x\right\}$, where $X_{\infty}$ is the buffer occupancy when the buffer capacity is infinite and the system is stable (i.e. $\mathbb{E}(\Lambda)<0$ ). For large $x$, large deviations techniques can be used to approximate this latter probability.

In this paper we study the quantity $\pi_{\text {loss }}$ defined by (1) by considering congestion periods on a link equipped with a finite buffer. For this purpose, we develop a method of computing the quantity $\pi_{\text {loss }}$ when the input process is modulated by a Markov process. More specifically, we assume that the input rate is of the form $\chi_{\varphi(t)}$, where the phase process $(\varphi(t))$ is a Markov process with a finite state space $S$ and $\chi$ is some real-valued function from $S \rightarrow \mathbb{R}$. We specifically establish the law of the volume of fluid lost during an overflow period (i.e. a time period during which the occupancy of the buffer is $x$ ) as well as the duration of such a period.

The model considered is a stochastic fluid model and in particular a Markov modulated fluid queue. These models have been widely used in several domains of system performance evaluation such as manufacturing systems, communication networks, risk processes in insurance, and environmental problems; see, for example, [6] and the references therein. The transient and stationary behaviors of these models have been extensively studied and numerous performance metrics with their corresponding numerical algorithms have been proposed and derived in the literature; see [8] for a long list of references on the subject.

Hitting probabilities for stochastic fluid models have been analyzed in [2], [4], and [6]; the relevant algorithms have been developed in [3] and [5]. In [14], the authors studied these hitting probabilities together with the maximum peak and the minimum buffer level observed within a busy period of the queue. Here, we extend the analysis provided in [14] by considering the loss of fluid occurring in finite buffer fluid queues. It is worth noting that the authors of [10] considered similar performance metrics via the use of Laplace transforms. The key difference with that paper is that we compute the distribution of the random variables under consideration instead of their Laplace transforms. In particular, we compute the joint distributions of the congestion durations and the lost volumes in successive congestion periods in the same busy period. Finally, we prove that the total congestion duration and lost volume in a typical stationary busy period have phase type distributions.

The organization of this paper is as follows. In Section 2 we describe the model under consideration. In Section 3 we compute the distribution of the duration and the volume of lost information of the first congestion event; we also study the state by which the successive idle 
and busy periods start. In Section 4 we compute the joint distribution of all the loss periods in a busy period and the joint distribution of all the loss volumes in a busy period. These results are used in Section 5 to compute the total length of congestion periods and the total volume of information lost in a busy period. This enables us to compute the loss ratio $\pi_{\text {loss. }}$. Some concluding remarks are presented in Section 6.

\section{Model description}

\subsection{Notation}

We consider a fluid queue with a finite buffer of capacity denoted by $x$. The input and service rates are controlled by a homogeneous Markov process $\varphi=(\varphi(t), t \geq 0)$ on the finite state space $S$ with infinitesimal generator $\boldsymbol{T}$ and initial probability distribution $\boldsymbol{\alpha}$. Let $X(t)$ denote the amount of fluid in the buffer at time $t$. The process $((X(t), \varphi(t)), t \geq 0)$ forms a Markov process having a pair of continuous and discrete states.

Let $\chi_{i}$ be the input rate and $\eta_{i}$ be the service rate of the queue when the Markov process $\varphi$ is in state $i$. We denote by $r_{i}$ the net input rate of state $i$, i.e. $r_{i}=\chi_{i}-\eta_{i}$ and we define the diagonal matrix of net input rates $\boldsymbol{R}=\operatorname{diag}\left(r_{i}, i \in S\right)$. We denote by $S^{0}, S^{-}$and $S^{+}$the subsets of states $i \in S$ such that $r_{i}=0, r_{i}<0$ and $r_{i}>0$, respectively. In the same way, we denote by $\boldsymbol{R}_{0}, \boldsymbol{R}_{-}$, and $\boldsymbol{R}_{+}$the diagonal matrices $\boldsymbol{R}_{0}=\operatorname{diag}\left(r_{i}, i \in S^{0}\right), \boldsymbol{R}_{-}=\operatorname{diag}\left(-r_{i}, i \in S^{-}\right)$, and $\boldsymbol{R}_{+}=\operatorname{diag}\left(r_{i}, i \in S^{+}\right)$. We clearly have $\boldsymbol{R}_{0}=0$, the null matrix. The numbers of states in $S^{0}, S^{+}$, and $S^{-}$are denoted by $n_{0}, n_{-}$, and $n_{+}$, respectively. We have $\Lambda(t)=r_{\varphi(t)}$.

By using this partition of states, we decompose the transition matrix $\boldsymbol{T}$ as

$$
\boldsymbol{T}=\left(\begin{array}{ccc}
\boldsymbol{T}_{--} & \boldsymbol{T}_{-0} & \boldsymbol{T}_{-+} \\
\boldsymbol{T}_{0-} & \boldsymbol{T}_{00} & \boldsymbol{T}_{0+} \\
\boldsymbol{T}_{+-} & \boldsymbol{T}_{+0} & \boldsymbol{T}_{++}
\end{array}\right)
$$

We suppose that the Markov chain $(\varphi(t))$ has a stationary regime and that $\mathbb{E}\left(r_{\varphi(t)}\right)<0$ so that the busy periods of the queue have a finite mean value.

\subsection{Congestion metrics}

Assume that the buffer is initially empty $(X(0)=0)$ and that $\varphi(0) \in S^{+}$, so that a busy period starts at time 0 . For $u \in[0, x]$, let $\theta(u)$ be the first positive time at which the fluid reaches level $u$, i.e. $\theta(u)=\inf \{t>0 \mid X(t)=u\}$.

Let us characterize congestion by the distribution of the two random variables:

- the total time $\tau(x)$ during which the buffer is full in a busy period;

- the total quantity of fluid $V(x)$ lost in a busy period.

The random variables $\tau(x)$ and $V(x)$ are formally defined by

$$
\tau(x)=\int_{0}^{\theta(0)} \mathbf{1}_{\{X(t)=x\}} \mathrm{d} t, \quad V(x)=\int_{0}^{\theta(0)} r_{\varphi(t)} \mathbf{1}_{\{X(t)=x\}} \mathrm{d} t .
$$

Note that $X(t)=x$ implies that $\varphi(t) \in S^{+} \cup S^{0}$ and when $\varphi(t) \in S^{0}, r_{\varphi(t)}=0$.

We consider the successive periods when the buffer capacity $x$ is reached by process $(X(t))$. We denote by $K$ the number of such periods occurring during a busy period of the buffer. Clearly, $K$ is an integer-valued random variable and if $K=0$, which means that process never reaches level $x$ during the busy period, then we have $V(x)=0$. We thus consider the case when $K \geq 1$. 


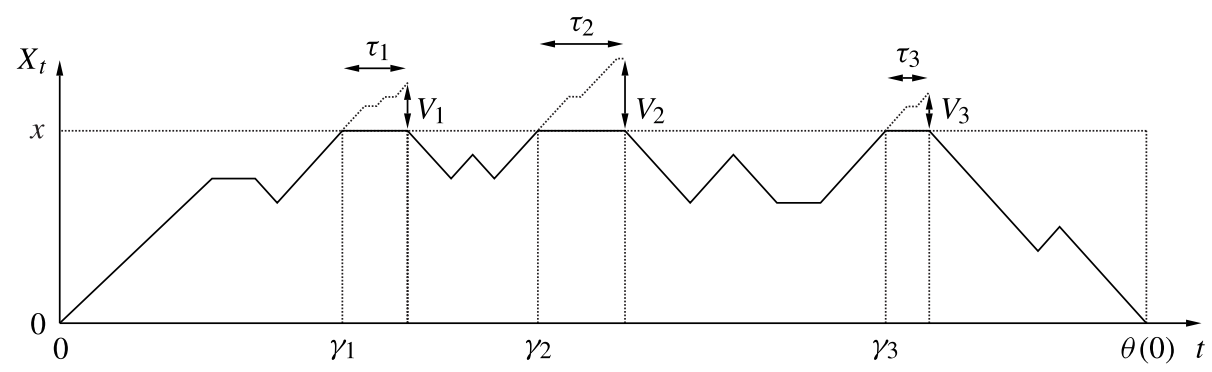

FIGURE 1: A typical busy period.

For every $k \geq 1$, we denote by $\gamma_{k}$ the $k$ th instant at which $X\left(\gamma_{k}\right)=x$ and $X\left(\gamma_{k}^{-}\right)<x$. We also denote by $\tau_{k}(x)$ the duration of the $k$ th period during which the fluid level is equal to $x$. More precisely, if we set $\gamma_{0}=0$ and $\tau_{0}(x)=0$, we have, for every $k \geq 1$,

$$
\begin{gathered}
\gamma_{k}=\inf \left\{t \in\left(\gamma_{k-1}+\tau_{k-1}(x), \theta(0)\right) \mid X(t)=x\right\}, \\
\tau_{k}(x)=\inf \left\{t>\gamma_{k} \mid X(t)<x\right\}-\gamma_{k} .
\end{gathered}
$$

We have $\gamma_{1}=\theta(x)$. In addition, $\gamma_{k}$ also depends on $x$ but we do not mention this dependence to simplify the notation. When $K \geq 1$, for $k \in\{1, \ldots, K\}$, we denote by $V_{k}(x)$ the volume of fluid lost during period $\tau_{k}(x)$. This volume is easily expressed as

$$
V_{k}(x)=\int_{\gamma_{k}}^{\tau_{k}(x)+\gamma_{k}} r_{\varphi(t)} \mathrm{d} t
$$

and, we have

$$
\tau(x)=\sum_{k=1}^{K} \tau_{k}(x), \quad V(x)=\sum_{k=1}^{K} V_{k}(x) .
$$

The above variables are shown in Figure 1 for the $K=3$ case. To simplify the figure, we have chosen the nonzero effective input rates equal to -1 or 1 . In Figure 1 , the successive volumes of fluid lost during the busy period is shown above the dotted line at $X(t)=x$.

Note that, for every $k \geq 1$ and for every $j \in S^{+}$, we have

$$
\varphi\left(\gamma_{k}\right)=j \quad \Longrightarrow \quad \varphi\left(\gamma_{k}\right) \in S^{+} \Longleftrightarrow K \geq k .
$$

\subsection{Reduction of the state space}

Let us introduce the $\left(n_{-}+n_{+}, n_{-}+n_{+}\right)$matrix

$$
\boldsymbol{Q}=\left(\begin{array}{ll}
\boldsymbol{Q}_{--} & \boldsymbol{Q}_{-+} \\
\boldsymbol{Q}_{+-} & \boldsymbol{Q}_{++}
\end{array}\right)
$$

where $\boldsymbol{Q}_{--}=\boldsymbol{R}_{-}^{-1}\left(\boldsymbol{T}_{--}-\boldsymbol{T}_{-0} \boldsymbol{T}_{00}^{-1} \boldsymbol{T}_{0-}\right), \boldsymbol{Q}_{-+}=\boldsymbol{R}_{-}^{-1}\left(\boldsymbol{T}_{-+}-\boldsymbol{T}_{-0} \boldsymbol{T}_{00}^{-1} \boldsymbol{T}_{0+}\right), \boldsymbol{Q}_{+-}=$ $\boldsymbol{R}_{+}^{-1}\left(\boldsymbol{T}_{+-}-\boldsymbol{T}_{+0} \boldsymbol{T}_{00}^{-1} \boldsymbol{T}_{0-}\right)$, and $\boldsymbol{Q}_{++}=\boldsymbol{R}_{+}^{-1}\left(\boldsymbol{T}_{++}-\boldsymbol{T}_{+0} \boldsymbol{T}_{00}^{-1} \boldsymbol{T}_{0+}\right)$. As shown in [4] and [12], when considering quantities such as hitting probabilities and accumulated rewards during sojourn times in $S^{+} \cup S^{0}$, the fluid queue with parameters $(\boldsymbol{T}, \boldsymbol{R})$ is equivalent to the fluid queue with parameters $(\boldsymbol{Q}, \boldsymbol{C})$, where $\boldsymbol{C}$ is the $\left(n_{-}+n_{+}, n_{-}+n_{+}\right)$matrix

$$
\boldsymbol{C}=\left(\begin{array}{cc}
-\boldsymbol{I} & 0 \\
0 & \boldsymbol{I}
\end{array}\right)
$$


and $\boldsymbol{I}$ is the identity matrix whose dimension is specified by the context of its use. This transformation was also suggested in [1] and [11].

The distribution of $\tau_{k}(x)$, given that the phase at time $\gamma_{k}$ is $j \in S^{+}$, is given by

$$
\mathbb{P}\left\{\tau_{k}(x)>t \mid \varphi\left(\gamma_{k}\right)=j\right\}=\left(\mathrm{e}^{A t} \mathbb{1}\right)_{j},
$$

where $\boldsymbol{A}$ is the $\left(n_{+}+n_{0}, n_{+}+n_{0}\right)$ matrix defined by

$$
\boldsymbol{A}=\left(\begin{array}{ll}
\boldsymbol{T}_{00} & \boldsymbol{T}_{0+} \\
\boldsymbol{T}_{+0} & \boldsymbol{T}_{++}
\end{array}\right)
$$

and 1 is the column vector with all its entries equal to 1 , its dimension being defined by the context. Since $j \in S^{+}$, we only need the entries of $\mathrm{e}^{A t} \|$ corresponding to the subset $S^{+}$, so we decompose vector $\mathrm{e}^{A t} \|$ with respect to subsets $S^{0}$ and $S^{+}$, to obtain

$$
\mathrm{e}^{A t} 1=\left(\begin{array}{c}
\left(\mathrm{e}^{A t} 1\right)^{0} \\
\left(\mathrm{e}^{A t} 1\right)^{+}
\end{array}\right)
$$

We then have

$$
\mathbb{P}\left\{\tau_{k}(x)>t \mid \varphi\left(\gamma_{k}\right)=j\right\}=\left(\mathrm{e}^{A t} 1\right)_{j}^{+} .
$$

Now, if we consider the volume of fluid $V_{k}(x)$ lost during period $\tau_{k}(x)$, given that the phase at time $\gamma_{k}$ is $j$, then $V_{k}(x)$ can also be seen as the accumulated reward during a sojourn of Markov chain $(\varphi(t))$ in subset $S^{0} \cup S^{+}$, starting in state $j \in S^{+}$. Using the results of [12], for $j \in S^{+}$, we obtain

$$
\mathbb{P}\left\{V_{k}(x)>v \mid \varphi\left(\gamma_{k}\right)=j\right\}=\left(\mathrm{e}^{\boldsymbol{Q}_{++} v} \|\right)_{j} .
$$

Thus, in the following, we will consider a fluid queue driven by a Markov chain $(\varphi(t))$ with state space $S=S^{-} \cup S^{+}$, infinitesimal generator $\boldsymbol{Q}$ and effective input rates given by matrix $\boldsymbol{C}$, i.e. equal to -1 or +1 .

\section{Distributions of $\tau_{1}(x)$ and $V_{1}(x)$}

\subsection{Matrix differential equation}

In order to determine the distribution of $\tau_{1}(x)$ and $V_{1}(x)$, we need the distribution of the Markov chain $(\varphi(t))$ at time $\gamma_{1}$. We introduce, for $i \in S^{+}$and $j \in S^{-}$,

$$
\Psi_{i, j}(x)=\mathbb{P}\{\varphi(\theta(0))=j, X(s)<x \text { for } 0 \leq s \leq \theta(0) \mid \varphi(0)=i, X(0)=0\}
$$

and, for $i \in S^{+}$and $j \in S^{+}, M_{i, j}(x)=\mathbb{P}\left\{\varphi\left(\gamma_{1}\right)=j \mid X(0)=0, \varphi(0)=i\right\}$. Let $\Psi(x)$ and $\boldsymbol{M}(x)$ denote the matrix with entries $\Psi_{i, j}(x)$ and $M_{i, j}(x)$, respectively. For all $i \in S^{+}$, $\sum_{j \in S^{-}} \Psi_{i, j}(x)$ is the probability that the process does not hit level $x$ in a busy period starting from state $i$ and an empty buffer and $\sum_{j \in S^{+}} M_{i, j}(x)$ is the probability of the complementary event. Hence, for all $i \in S^{+}, \sum_{j \in S^{-}} \Psi_{i, j}(x)+\sum_{j \in S^{+}} M_{i, j}(x)=1$. This can be written in matrix form as $\Psi(x) \mathbb{1}+\boldsymbol{M}(x) \mathbb{\|}=\mathbb{1}$, where we recall that the dimension of $\mathbb{1}$ is given by the context of its use.

Matrices $\boldsymbol{M}(x)$ and $\boldsymbol{\Psi}(x)$ are related to each other via a differential equation. As shown in [14, Corollary 3], matrix $\boldsymbol{\Psi}(x)$ satisfies the following integral equation:

$$
\boldsymbol{\Psi}(x)=\int_{0}^{x} \mathrm{e}^{\boldsymbol{Q}_{++} y} \boldsymbol{Q}_{+-} \mathrm{e}^{\boldsymbol{Q}_{--} y} \mathrm{~d} y+\int_{0}^{x} \mathrm{e}^{\boldsymbol{Q}_{++} y} \boldsymbol{\Psi}(x-y) \boldsymbol{Q}_{-+} \boldsymbol{\Psi}(x-y) \mathrm{e}^{\boldsymbol{Q}_{--} y} \mathrm{~d} y .
$$


From [14, Theorem 4], we have the following matrix differential Riccati equation:

$$
\boldsymbol{\Psi}^{\prime}(x)=\boldsymbol{Q}_{++} \boldsymbol{\Psi}(x)+\boldsymbol{\Psi}(x) \boldsymbol{Q}_{--}+\boldsymbol{\Psi}(x) \boldsymbol{Q}_{-+} \boldsymbol{\Psi}(x)+\boldsymbol{Q}_{+-}
$$

with $\boldsymbol{\Psi}(0)=0$. Matrix $\boldsymbol{M}(x)$ is then related to $\boldsymbol{\Psi}(x)$ by the following result.

Theorem 1. For every $x \geq 0$, we have

$$
\begin{gathered}
\boldsymbol{M}(x)=\mathrm{e}^{\boldsymbol{Q}_{++} x}+\int_{0}^{x} \mathrm{e}^{\boldsymbol{Q}_{++} y} \boldsymbol{\Psi}(x-y) \boldsymbol{Q}_{-+} \boldsymbol{M}(x-y) \mathrm{d} y, \\
\boldsymbol{M}^{\prime}(x)=\left(\boldsymbol{Q}_{++} \boldsymbol{\Psi}(x) \boldsymbol{Q}_{-+}\right) \boldsymbol{M}(x) .
\end{gathered}
$$

Proof. The proof of (6) follows the arguments developed in [4] or [14] and (7) is obtained by differentiating (6).

\subsection{Distributions of the random variables}

The distributions of $\tau_{1}(x)$ and $V_{1}(x)$ are given by the following theorem.

Theorem 2. For every $i \in S^{+}$and for all $v, t \geq 0$, we have

$$
\begin{gathered}
\mathbb{P}\left\{V_{1}(x)>v \mid \varphi(0)=i\right\}=\left(\boldsymbol{M}(x) \mathrm{e}^{\boldsymbol{Q}_{++} v} 1\right)_{i}, \\
\mathbb{P}\left\{\tau_{1}(x)>t \mid \varphi(0)=i\right\}=\left(\boldsymbol{M}(x)\left(\mathrm{e}^{\boldsymbol{A} t} 1\right)^{+}\right)_{i}, \quad \mathbb{P}\{K \geq 1 \mid \varphi(0)=i\}=(\boldsymbol{M}(x) \mathbb{1})_{i} .
\end{gathered}
$$

Proof. Using the Markov property, for every $i \in S^{+}$, we obtain

$$
\begin{aligned}
\mathbb{P}\left\{\tau_{1}(x)>t \mid \varphi(0)=i\right\} & =\sum_{j \in S^{+}} \mathbb{P}\left\{\tau_{1}(x)>t \mid \varphi\left(\gamma_{1}\right)=j\right\} \mathbb{P}\left\{\varphi\left(\gamma_{1}\right)=j \mid \varphi(0)=i\right\} \\
& =\sum_{j \in S^{+}} M_{i, j}(x)\left(\mathrm{e}^{\boldsymbol{A} t} 1\right)_{j}^{+} \\
& =\left(\boldsymbol{M}(x)\left(\mathrm{e}^{A t} 1\right)^{+}\right)_{i} .
\end{aligned}
$$

The same argument leads to (8). From (2), by definition of $M_{i, j}(x)$, we obtain

$$
\mathbb{P}\{K \geq 1 \mid \varphi(0)=i\}=\mathbb{P}\left\{\varphi\left(\gamma_{1}\right) \in S^{+} \mid \varphi(0)=i\right\}=(\boldsymbol{M}(x) \mathbb{1})_{i} .
$$

In the next section, we study the distributions of the state initiating the successive idle and busy periods of the buffer.

\subsection{Sequences of idle and busy periods}

We decompose the initial probability distribution $\boldsymbol{\alpha}$ of Markov chain $(\varphi(t))$ through the partition $S^{-}, S^{0}, S^{+}$, by writing $\boldsymbol{\alpha}=\left(\boldsymbol{\alpha}^{-}, \boldsymbol{\alpha}^{0}, \boldsymbol{\alpha}^{+}\right)$. Recall that we suppose that the buffer is initially empty, i.e. $X(0)=0$ with probability 1 . For every $n \geq 1$, we denote by $\mathrm{SI}_{n}$ the state or the phase by which the $n$th idle period starts and we denote by $\mathrm{SB}_{n}$ the state or the phase by which the $n$th busy period starts. We then have $\mathrm{SI}_{1} \in S^{-} \cup S^{0}$ if $\boldsymbol{\alpha}^{0} \neq 0, \mathrm{SI}_{n} \in S^{-}$for $n \geq 2$, and $\mathrm{SB}_{n} \in S^{+}$. We denote respectively by $\boldsymbol{v}_{n}$ and $\boldsymbol{w}_{n}$ the probability distributions of $\mathrm{SI}_{n}$ and $\mathrm{SB}_{n}$, i.e.

$$
\boldsymbol{w}_{n}=\left(\mathbb{P}\left\{\mathrm{SB}_{n}=j\right\}, j \in S^{+}\right), \quad \boldsymbol{v}_{1}=\left(\mathbb{P}\left\{\mathrm{SI}_{1}=j\right\}, j \in S^{-} \cup S^{0}\right) .
$$


For $n \geq 2$,

$$
\boldsymbol{v}_{n}=\left(\mathbb{P}\left\{\mathrm{SI}_{n}=j\right\}, j \in S^{-}\right) .
$$

For every $i \in S^{+}$and $j \in S^{-}$, we denote by $\Gamma_{i, j}(x)$ the probability that a busy period ends in phase $j$ given that it starts in phase $i$, i.e.

$$
\Gamma_{i, j}(x)=\mathbb{P}\{\varphi(\theta(0))=j \mid \varphi(0)=i\}
$$

and we denote by $\Gamma(x)$ the $\left(n_{+}, n_{-}\right)$matrix with components $\Gamma_{i, j}(x)$. It was shown in [14] that $\Gamma(x)$ is the unique solution to the matrix differential Riccati equation $\Gamma^{\prime}(x)=Q_{++} \Gamma(x)+$ $\boldsymbol{\Gamma}(x) \boldsymbol{Q}_{--}+\boldsymbol{\Gamma}(x) \boldsymbol{Q}_{-+} \boldsymbol{\Gamma}(x)+\boldsymbol{Q}_{+-}$with $\boldsymbol{\Gamma}(0)=\left(-\boldsymbol{Q}_{++}\right)^{-1} \boldsymbol{Q}_{+-}$. We remark that for every $x \geq 0$, we have $\Gamma(x) \|=1$. Using the results of [12], we have

$$
\boldsymbol{v}_{1}=\left(\boldsymbol{\alpha}^{-}+\boldsymbol{\alpha}^{+} \boldsymbol{\Gamma}(x), \boldsymbol{\alpha}^{0}\right), \quad \boldsymbol{w}_{1}=\boldsymbol{\alpha}^{+}+\left(\boldsymbol{\alpha}^{-}, \boldsymbol{\alpha}^{0}\right)\left(-\boldsymbol{A}^{\prime}\right)^{-1} \boldsymbol{B}^{\prime},
$$

where matrices $\boldsymbol{A}^{\prime}$ and $\boldsymbol{B}^{\prime}$ are defined by

$$
\boldsymbol{A}^{\prime}=\left(\begin{array}{cc}
\boldsymbol{T}_{--} & \boldsymbol{T}_{-0} \\
\boldsymbol{T}_{0-} & \boldsymbol{T}_{00}
\end{array}\right), \quad \boldsymbol{B}^{\prime}=\left(\begin{array}{l}
\boldsymbol{T}_{-+} \\
\boldsymbol{T}_{0+}
\end{array}\right) .
$$

Defining $\boldsymbol{Q}_{00}=\boldsymbol{T}_{00}-\boldsymbol{T}_{0-} \boldsymbol{T}_{--}^{-1} \boldsymbol{T}_{-0}$ and $\boldsymbol{Q}_{0+}=\boldsymbol{T}_{0+}-\boldsymbol{T}_{0-} \boldsymbol{T}_{--}^{-1} \boldsymbol{T}_{-+}$, we have

$$
\left(-\boldsymbol{A}^{\prime}\right)^{-1}=\left(\begin{array}{cc}
\left(-\boldsymbol{Q}_{--}\right)^{-1} \boldsymbol{R}_{-}^{-1} & \left(-\boldsymbol{Q}_{--}\right)^{-1} \boldsymbol{R}_{-}^{-1} \boldsymbol{T}_{-0}\left(-\boldsymbol{T}_{00}\right)^{-1} \\
\left(-\boldsymbol{Q}_{00}\right)^{-1} \boldsymbol{T}_{0-}\left(-\boldsymbol{T}_{--}\right)^{-1} & \left(-\boldsymbol{Q}_{00}\right)^{-1}
\end{array}\right)
$$

and

$$
\left(-\boldsymbol{A}^{\prime}\right)^{-1} \boldsymbol{B}^{\prime}=\left(\begin{array}{cc}
\left(-\boldsymbol{Q}_{--}\right)^{-1} & \boldsymbol{Q}_{-+} \\
\left(-\boldsymbol{Q}_{00}\right)^{-1} & \boldsymbol{Q}_{0+}
\end{array}\right)
$$

which leads to

$$
\boldsymbol{w}_{1}=\boldsymbol{\alpha}^{+}+\boldsymbol{\alpha}^{-}\left(-\boldsymbol{Q}_{--}\right)^{-1} \boldsymbol{Q}_{-+}+\boldsymbol{\alpha}^{0}\left(-\boldsymbol{Q}_{00}\right)^{-1} \boldsymbol{Q}_{0+} .
$$

In the following theorem, we provide the expressions of $\boldsymbol{v}_{n}$ and $\boldsymbol{w}_{n}$ for $n \geq 2$.

Theorem 3. For every $n \geq 2$, we have

$$
\begin{aligned}
\boldsymbol{v}_{n} & =\left(\boldsymbol{\alpha}^{-}+\boldsymbol{\alpha}^{+} \boldsymbol{\Gamma}(x)\right) \mathcal{L}^{n-1}(x)+\boldsymbol{\alpha}^{0}\left(-\boldsymbol{Q}_{00}\right)^{-1} \boldsymbol{Q}_{0+} \boldsymbol{\Gamma}(x) \mathcal{L}^{n-2}(x), \\
\boldsymbol{w}_{n} & =\left(\boldsymbol{\alpha}^{+}+\boldsymbol{\alpha}^{-}\left(-\boldsymbol{Q}_{--}\right)^{-1} \boldsymbol{Q}_{-+}+\boldsymbol{\alpha}^{0}\left(-\boldsymbol{Q}_{00}\right)^{-1} \boldsymbol{Q}_{0+}\right) \mathcal{K}^{n-1}(x),
\end{aligned}
$$

where matrices $\mathcal{L}(x)$ and $\mathcal{K}(x)$ are given by

$$
\mathcal{L}(x)=\left(-\boldsymbol{Q}_{--}\right)^{-1} \boldsymbol{Q}_{-+} \boldsymbol{\Gamma}(x), \quad \mathcal{K}(x)=\boldsymbol{\Gamma}(x)\left(-\boldsymbol{Q}_{--}\right)^{-1} \boldsymbol{Q}_{-+} .
$$

Proof. For every $j \in S^{-}$, we have

$$
\mathbb{P}\left\{\mathrm{SI}_{2}=j\right\}=\sum_{i \in S^{-} \cup S^{0}} \mathbb{P}\left\{\mathrm{SI}_{1}=i\right\}\left[\left(-\boldsymbol{A}^{\prime}\right)^{-1} \boldsymbol{B}^{\prime} \boldsymbol{\Gamma}(x)\right]_{i, j},
$$

which gives, in matrix notation, using (9) and (11),

$$
\begin{aligned}
\boldsymbol{v}_{2} & =\boldsymbol{v}_{1}\left(-\boldsymbol{A}^{\prime}\right)^{-1} \boldsymbol{B}^{\prime} \boldsymbol{\Gamma}(x) \\
& =\left(\boldsymbol{\alpha}^{-}+\boldsymbol{\alpha}^{+} \boldsymbol{\Gamma}(x)\right)\left(-\boldsymbol{Q}_{--}\right)^{-1} \boldsymbol{Q}_{-+} \boldsymbol{\Gamma}(x)+\boldsymbol{\alpha}^{0}\left(-\boldsymbol{Q}_{00}\right)^{-1} \boldsymbol{Q}_{0+} \boldsymbol{\Gamma}(x) \\
& =\left(\boldsymbol{\alpha}^{-}+\boldsymbol{\alpha}^{+} \boldsymbol{\Gamma}(x)\right) \mathcal{L}(x)+\boldsymbol{\alpha}^{0}\left(-\boldsymbol{Q}_{00}\right)^{-1} \boldsymbol{Q}_{0+} \boldsymbol{\Gamma}(x) .
\end{aligned}
$$


For every $n \geq 3$ and $j \in S^{-}$, by homogeneity, we have

$$
\mathbb{P}\left\{\mathrm{SI}_{n}=j\right\}=\sum_{i \in S^{-}} \mathbb{P}\left\{\mathrm{SI}_{n-1}=i\right\}\left[\left(-\boldsymbol{A}^{\prime}\right)^{-1} \boldsymbol{B}^{\prime} \boldsymbol{\Gamma}(x)\right]_{i, j} .
$$

Thus, in matrix notation, again using (9) and (11), we obtain

$$
\boldsymbol{v}_{n}=\boldsymbol{v}_{n-1}\left(-\boldsymbol{Q}_{--}\right)^{-1} \boldsymbol{Q}_{-+} \boldsymbol{\Gamma}(x)=\boldsymbol{v}_{n-1} \mathcal{L}(x) .
$$

For $n \geq 2$ this leads to $\boldsymbol{v}_{n}=\boldsymbol{v}_{2} \mathfrak{L}^{n-2}(x)$, which is the desired result. In the same way, for every $n \geq 2$ and $j \in S^{+}$, using (11), we have

$$
\begin{aligned}
\mathbb{P}\left\{\mathrm{SB}_{n}=j\right\} & =\sum_{i \in S^{+}} \mathbb{P}\left\{\mathrm{SB}_{n-1}=i\right\} \sum_{\ell \in S^{-}} \Gamma_{i, \ell}(x)\left[\left(-\boldsymbol{A}^{\prime}\right)^{-1} \boldsymbol{B}^{\prime}\right]_{\ell, j} \\
& =\sum_{i \in S^{+}} \mathbb{P}\left\{\mathrm{SB}_{n-1}=i\right\}\left[\boldsymbol{\Gamma}(x)\left(-\boldsymbol{Q}_{--}\right)^{-1} \boldsymbol{Q}_{-+}\right]_{i, j} \\
& =\sum_{i \in S^{+}} \mathbb{P}\left\{\mathrm{SB}_{n-1}=i\right\} \mathcal{K}_{i, j}(x) .
\end{aligned}
$$

In matrix notation, using (12), we obtain $\boldsymbol{w}_{n}=\boldsymbol{w}_{n-1} \mathcal{K}(x)$, i.e. $\boldsymbol{w}_{n}=\boldsymbol{w}_{1} \mathcal{K}^{n-1}(x)$.

The Markov chain $(\varphi(t))$ being finite and irreducible, matrices $\mathcal{L}(x)$ and $\mathcal{K}(x)$, which are stochastic matrices are also irreducible and aperiodic. Thus, we have

$$
\lim _{n \rightarrow \infty} \boldsymbol{v}_{n}=\boldsymbol{\pi}^{-}, \quad \lim _{n \rightarrow \infty} \boldsymbol{w}_{n}=\boldsymbol{\pi}^{+},
$$

where $\pi^{-}$is a row vector of dimension $n_{-}$, which is the unique solution to the system $\pi^{-}=$ $\pi^{-} \mathcal{L}(x)$ with $\pi^{-} 1=1$ and $\pi^{+}$is a row vector of dimension $n_{+}$, which is the unique solution to the system $\pi^{+}=\pi^{+} \mathcal{K}(x)$ with $\pi^{+} \mathbb{\|}=1$.

In what follows we suppose that the queue is in the stationary regime, i.e. the distribution of $\varphi(0)$ is $\pi^{+}$. Thus, we have $\mathbb{P}\left\{\tau_{1}(x)>t\right\}=\pi^{+} \boldsymbol{M}(x)\left(\mathrm{e}^{\boldsymbol{A} t} 1\right)^{+}, \mathbb{P}\left\{V_{1}(x)>v\right\}=$ $\boldsymbol{\pi}^{+} \boldsymbol{M}(x) \mathrm{e}^{\boldsymbol{Q}_{++} v} 1$, and $\mathbb{P}\{K \geq 1\}=\boldsymbol{\pi}^{+} \boldsymbol{M}(x) 1$. The above result provides the distribution of the first congestion event. In the next section we compute the characteristics of several congestion events.

\section{Joint distributions of loss periods and loss volumes}

In this section we study the joint distribution of the times $\tau_{k}(x)$ and the joint distribution of the volumes $V_{k}(x)$. To deal with these distributions, we need to evaluate the probability $H_{i, j}(x)$, starting from level $x$ in phase $i \in S^{-}$, that the phase at the time of the first return to level $x$ is equal to $j$, without hitting level 0 . This probability can be interpreted as the symmetric probability of $\Psi(x)$ with respect to levels 0 and $x$. It is defined, for every $i \in S^{-}$and $j \in S^{+}$, by

$$
H_{i, j}(x)=\mathbb{P}\{\varphi(\theta(x))=j, X(s)>0 \text { for } 0 \leq s \leq \theta(x) \mid \varphi(0)=i, X(0)=x\} .
$$

Theorem 4. For every $x \geq 0$, we have

$$
\boldsymbol{H}(x)=\int_{0}^{x} \mathrm{e}^{\boldsymbol{Q}_{--y}} \boldsymbol{Q}_{-+} \mathrm{e}^{\boldsymbol{Q}_{++} y} \mathrm{~d} y+\int_{0}^{x} \mathrm{e}^{\boldsymbol{Q}_{--y}} \boldsymbol{H}(x-y) \boldsymbol{Q}_{+-} \boldsymbol{H}(x-y) \mathrm{e}^{\boldsymbol{Q}_{++} y} \mathrm{~d} y .
$$


Proof. The proof is quite similar to the proof leading to (4). It can also be seen as a particular case of the way in which [14, Equation (12)] was obtained since here we do not consider the paths hitting level 0 .

In the same way that we obtained (5), matrix $\boldsymbol{H}(x)$ satisfies $\boldsymbol{H}(0)=0$ and

$$
\boldsymbol{H}^{\prime}(x)=\boldsymbol{Q}_{--} \boldsymbol{H}(x)+\boldsymbol{H}(x) \boldsymbol{Q}_{++}+\boldsymbol{H}(x) \boldsymbol{Q}_{+-} \boldsymbol{H}(x)+\boldsymbol{Q}_{-+} .
$$

Note that, by the homogeneity of $\varphi$, we also have, for every $i \in S^{-}, j \in S^{+}$, and $k \geq 1$,

$$
H_{i, j}(x)=\mathbb{P}\left\{\varphi\left(\gamma_{k+1}\right)=j \mid \varphi\left(\gamma_{k}+\tau_{k}\right)=\ell\right\} .
$$

This relation is used to prove the next lemma. We introduce the matrix $\boldsymbol{B}$ defined by

$$
\boldsymbol{B}=\left(\begin{array}{c}
\boldsymbol{T}_{+-} \\
\boldsymbol{T}_{0-}
\end{array}\right) .
$$

Lemma 1. For every $k \geq 1, v \geq 0$, and $i, j \in S^{+}$, we have

$$
\begin{gathered}
\mathbb{P}\left\{V_{k}(x)>v, \varphi\left(\gamma_{k+1}\right)=j \mid \varphi\left(\gamma_{k}\right)=i\right\}=\left(\mathrm{e}^{\boldsymbol{Q}_{++} v}\left(-\boldsymbol{Q}_{++}\right)^{-1} \boldsymbol{Q}_{+-} \boldsymbol{H}(x)\right)_{i, j}, \\
\mathbb{P}\left\{\tau_{k}(x)>t, \varphi\left(\gamma_{k+1}\right)=j \mid \varphi\left(\gamma_{k}\right)=i\right\}=\left(\left[\mathrm{e}^{\boldsymbol{A} t_{\ell}}(-\boldsymbol{A})^{-1} \boldsymbol{B}\right]_{+-} \boldsymbol{H}(x)\right)_{i, j},
\end{gathered}
$$

where matrix $\left[\mathrm{e}^{\boldsymbol{A} t_{\ell}}(-\boldsymbol{A})^{-1} \boldsymbol{B}\right]_{+-}$is the block $\left(n_{+}, n_{-}\right)$of matrix $\mathrm{e}^{\boldsymbol{A} t_{\ell}}(-\boldsymbol{A})^{-1} \boldsymbol{B}$.

Proof. The homogeneity of Markov chain $\varphi$ implies that, for every $k \geq 1$,

$$
\mathbb{P}\left\{V_{k}(x)>v, \varphi\left(\gamma_{k+1}\right)=j \mid \varphi\left(\gamma_{k}\right)=i\right\}=\mathbb{P}\left\{V_{1}(x)>v, \varphi\left(\gamma_{2}\right)=j \mid \varphi\left(\gamma_{1}\right)=i\right\},
$$

so we set $k=1$. Note that the entry $(i, \ell)$ of matrix e $\boldsymbol{Q}_{++}^{v}\left(-\boldsymbol{Q}_{++}\right)^{-1} \boldsymbol{Q}_{+-}$is (see [12]) the probability, starting from $i \in S^{+}$, that the accumulated reward during a sojourn of $\varphi$ in $S^{+} \cup S^{0}$ is greater than $v$ and that the first state of $S^{-}$visited after this sojourn is $\ell$. Conditioning on the phase at time $\gamma_{1}+\tau_{1}$ and using the Markov property, we obtain

$$
\begin{aligned}
& \mathbb{P}\left\{\varphi\left(\gamma_{2}\right)=j, V_{1}(x)>v \mid \varphi\left(\gamma_{1}\right)=i\right\} \\
& \quad=\sum_{\ell \in S^{-}} \mathbb{P}\left\{\varphi\left(\gamma_{2}\right)=j \mid \varphi\left(\gamma_{1}+\tau_{1}\right)=\ell\right\} \mathbb{P}\left\{\varphi\left(\gamma_{1}+\tau_{1}\right)=\ell, V_{1}(x)>v \mid \varphi\left(\gamma_{1}\right)=i\right\} \\
& =\sum_{\ell \in S^{-}} H_{\ell, j}\left(\mathrm{e}^{\boldsymbol{Q}_{++} v}\left(-\boldsymbol{Q}_{++}\right)^{-1} \boldsymbol{Q}_{+-}\right)_{i, \ell} \\
& =\left(\mathrm{e}^{\boldsymbol{Q}_{++} v}\left(-\boldsymbol{Q}_{++}\right)^{-1} \boldsymbol{Q}_{+-} \boldsymbol{H}(x)\right)_{i, j} .
\end{aligned}
$$

To obtain the second relation, we proceed in the same way noting that the entry $(i, \ell)$ of matrix $\left[\mathrm{e}^{A t_{\ell}}(-\boldsymbol{A})^{-1} \boldsymbol{B}\right]_{+-}$is (see [12]) the probability, starting from $i \in S^{+}$, that a sojourn of $\varphi$ in $S^{+} \cup S^{0}$ is greater than $v$ and that the first state of $S^{-}$visited after this sojourn is $\ell$.

We remark that we have the following relations:

$$
\left[(-\boldsymbol{A})^{-1} \boldsymbol{B}\right]_{+-}=\left(-\boldsymbol{Q}_{++}\right)^{-1} \boldsymbol{Q}_{+-}, \quad\left(-\boldsymbol{Q}_{++}\right)^{-1} \boldsymbol{Q}_{+-} \mathbb{1}=\mathbb{1} .
$$

Theorem 5. For every $k \geq 1$ and $v_{1}, \ldots, v_{k} \geq 0$, we have

$$
\begin{aligned}
& \mathbb{P}\left\{V_{1}(x)>v_{1}, V_{2}(x)>v_{2}, \ldots, V_{k}(x)>v_{k}\right\} \\
& \quad=\pi^{+} \boldsymbol{M}(x)\left(\prod_{\ell=1}^{k-1} \mathrm{e}^{\boldsymbol{Q}_{++} v_{\ell}}\left(-\boldsymbol{Q}_{++}\right)^{-1} \boldsymbol{Q}_{+-} \boldsymbol{H}(x)\right) \mathrm{e}^{\boldsymbol{Q}_{++} v_{k}} 1 .
\end{aligned}
$$


Proof. Note that, by convention, the product is equal to 1 for $k=1$. Let us define $F_{i, j}(k)$, for every $k \geq 1$ and $i, j \in S^{+}$, by

$$
F_{i, j}(k)=\mathbb{P}\left\{V_{1}(x)>v_{1}, \ldots, V_{k}(x)>v_{k}, \varphi\left(\gamma_{k+1}\right)=j \mid \varphi(0)=i\right\} .
$$

Conditioning on $\varphi\left(\gamma_{k}\right)$ and using the Markov property, we have

$$
\begin{aligned}
F_{i, j}(k) & =\sum_{\ell \in S^{+}} \mathbb{P}\left\{V_{1}(x)>v_{1}, \ldots, V_{k}(x)>v_{k}, \varphi\left(\gamma_{k+1}\right)=j, \varphi\left(\gamma_{k}\right)=\ell \mid \varphi(0)=i\right\} \\
& =\sum_{\ell \in S^{+}} F_{i, \ell}(k-1) \mathbb{P}\left\{V_{k}(x)>v_{k}, \varphi\left(\gamma_{k+1}\right)=j \mid \varphi\left(\gamma_{k}\right)=\ell\right\} .
\end{aligned}
$$

Denoting by $\boldsymbol{F}(k)$ the matrix $\left[F_{i, j}(k)\right]_{i, j \in S^{+}}$and using Lemma 1, we obtain

$$
\boldsymbol{F}(k)=\boldsymbol{F}(k-1) \mathrm{e}^{\boldsymbol{Q}_{++} v_{k}}\left(-\boldsymbol{Q}_{++}\right)^{-1} \boldsymbol{Q}_{+-} \boldsymbol{H}(x),
$$

and, thus,

$$
\boldsymbol{F}(k)=\boldsymbol{F}(1) \prod_{\ell=2}^{k} \mathrm{e}^{\boldsymbol{Q}_{++} v_{\ell}}\left(-\boldsymbol{Q}_{++}\right)^{-1} \boldsymbol{Q}_{+-} \boldsymbol{H}(x) .
$$

Matrix $\boldsymbol{F}(1)$ is obtained in the same way, using the Markov property and Lemma 1, as

$$
\begin{aligned}
F_{i, j}(1) & =\sum_{\ell \in S^{+}} \mathbb{P}\left\{V_{1}(x)>v_{1}, \varphi\left(\gamma_{2}\right)=j, \varphi\left(\gamma_{1}\right)=\ell \mid \varphi(0)=i\right\} \\
& =\sum_{\ell \in S^{+}} \mathbb{P}\left\{\varphi\left(\gamma_{1}\right)=\ell \mid \varphi(0)=i\right\} \mathbb{P}\left\{V_{1}(x)>v_{1}, \varphi\left(\gamma_{2}\right)=j \mid \varphi\left(\gamma_{1}\right)=\ell\right\} \\
& =\sum_{\ell \in S^{+}} M_{i, \ell}(x)\left[\mathrm{e}^{\boldsymbol{Q}_{++} v_{1}}\left(-\boldsymbol{Q}_{++}\right)^{-1} \boldsymbol{Q}_{+-} \boldsymbol{H}(x)\right]_{\ell, j} .
\end{aligned}
$$

Finally, we have

$$
\boldsymbol{F}(k)=\boldsymbol{M}(x) \prod_{\ell=1}^{k} \mathrm{e}^{\boldsymbol{Q}_{++} v_{\ell}}\left(-\boldsymbol{Q}_{++}\right)^{-1} \boldsymbol{Q}_{+-} \boldsymbol{H}(x) .
$$

To obtain the joint distribution of $V_{1}(x), \ldots, V_{k}(x)$, we use (3), the previous relation, and the Markov property, by writing

$$
\begin{aligned}
& \mathbb{P}\left\{V_{1}(x)>v_{1}, V_{2}(x)>v_{2}, \ldots, V_{k}(x)>v_{k} \mid \varphi(0)=i\right\} \\
& =\sum_{j \in S^{+}} \mathbb{P}\left\{V_{1}(x)>v_{1}, \ldots, V_{k-1}(x)>v_{k-1}, \varphi\left(\gamma_{k}\right)=j, V_{k}(x)>v_{k} \mid \varphi(0)=i\right\} \\
& =\sum_{j \in S^{+}} F_{i, j}(k-1) \mathbb{P}\left\{V_{k}(x)>v_{k} \mid \varphi\left(\gamma_{k}\right)=j\right\} \\
& =\sum_{j \in S^{+}} F_{i, j}(k-1)\left(\mathrm{e}^{\boldsymbol{Q}_{++} v_{k}} 1\right)_{j} \\
& =\left[F(k-1) \mathrm{e}^{\boldsymbol{Q}_{++} v_{k}} \mathbb{1}\right]_{i} .
\end{aligned}
$$

Since the distribution of $\varphi(0)$ is $\pi^{+}$, by unconditioning, we obtain

$$
\mathbb{P}\left\{V_{1}(x)>v_{1}, V_{2}(x)>v_{2}, \ldots, V_{k}(x)>v_{k}\right\}=\pi^{+} \boldsymbol{F}(k-1) \mathrm{e}^{\boldsymbol{Q}_{++} v_{k}} 1,
$$

which completes the proof. 
Similar arguments are used to obtain the joint distribution of the $\tau_{k}(x)$.

Theorem 6. For every $k \geq 1$ and $t_{1}, \ldots, t_{k} \geq 0$, we have

$$
\begin{aligned}
& \mathbb{P}\left\{\tau_{1}(x)>t_{1}, \tau_{2}(x)>t_{2}, \ldots, \tau_{k}(x)>t_{k}\right\} \\
& \quad=\pi^{+} \boldsymbol{M}(x)\left(\prod_{\ell=1}^{k-1}\left(\mathrm{e}^{\boldsymbol{A} t_{\ell}}(-\boldsymbol{A})^{-1} \boldsymbol{B}\right)_{+-} \boldsymbol{H}(x)\right)\left(\mathrm{e}^{A t_{k}} 1\right)^{+} .
\end{aligned}
$$

Note that the joint distribution of the $\tau_{k}$, given by (15), can be written as

$$
\mathbb{P}\left\{\tau_{1}(x)>t_{1}, \ldots, \tau_{k}(x)>t_{k}\right\}=\boldsymbol{a}(x)\left(\prod_{\ell=1}^{k-1} \mathrm{e}^{\boldsymbol{A} t_{\ell}}(-\boldsymbol{A})^{-1} \boldsymbol{B} \boldsymbol{R}(x)\right) \mathrm{e}^{\boldsymbol{A} t_{k}} \mathbb{1},
$$

where $\boldsymbol{a}(x)$ is the row vector defined by $\boldsymbol{a}(x)=\left(\boldsymbol{\pi}^{+} \boldsymbol{M}(x) \mathbf{0}\right), \mathbf{0}$ being here the null row vector of dimension $n_{0}$, and $\boldsymbol{R}(x)$ is the matrix defined by $\boldsymbol{R}(x)=(\boldsymbol{H}(x) \quad \mathbf{0}), \mathbf{0}$ being here the $\left(n_{-}, n_{0}\right)$ null matrix.

Corollary 1. For every $k \geq 1$ and $v, t \geq 0$, we have

$$
\begin{aligned}
\mathbb{P}\left\{V_{k}(x)>v\right\} & =\pi_{+} \boldsymbol{M}(x)\left(\left(-\boldsymbol{Q}_{++}\right)^{-1} \boldsymbol{Q}_{+-} \boldsymbol{H}(x)\right)^{k-1} \mathrm{e}^{\boldsymbol{Q}_{++} v} 1, \\
\mathbb{P}\left\{\tau_{k}(x)>t\right\} & =\pi_{+} \boldsymbol{M}(x)\left(\left(-\boldsymbol{Q}_{++}\right)^{-1} \boldsymbol{Q}_{+-} \boldsymbol{H}(x)\right)^{k-1}\left(\mathrm{e}^{A t} 1\right)^{+} .
\end{aligned}
$$

Proof. To obtain (17), we use $v_{1}=\cdots=v_{k-1}=0$ and $v_{k}=v$ in (14). To obtain (18), we use $t_{1}=\cdots=t_{k-1}=0$ and $t_{k}=t$ in (15) and observe that $\left((-\boldsymbol{A})^{-1} \boldsymbol{B}\right)_{+-}=$ $\left(-\boldsymbol{Q}_{++}\right)^{-1} \boldsymbol{Q}_{+-}$.

Corollary 2. For every $k \geq 0$, we have

$$
\mathbb{P}\{K>k\}=\boldsymbol{\pi}^{+} \boldsymbol{M}(x)\left(\left(-\boldsymbol{Q}_{++}\right)^{-1} \boldsymbol{Q}_{+-} \boldsymbol{H}(x)\right)^{k} 1 .
$$

Proof. For every $k \geq 0$, we have

$$
V_{k+1}(x)>0 \Longleftrightarrow \tau_{k+1}(x)>0 \Longleftrightarrow K>k .
$$

Thus, using either (17) or (18), we have $\mathbb{P}\{K>k\}=\boldsymbol{\pi}^{+} \boldsymbol{M}(x)\left(\left(-\boldsymbol{Q}_{++}\right)^{-1} \boldsymbol{Q}_{+-} \boldsymbol{H}(x)\right)^{k} 1$.

As in the joint distribution of the $\tau_{k}$, obtained in (16), the distribution of $K$, given by (19), can be written as

$$
\mathbb{P}\{K>k\}=\boldsymbol{a}(x)\left((-\boldsymbol{A})^{-1} \boldsymbol{B} \boldsymbol{R}(x)\right) \mathbb{1} .
$$

\section{Total duration of losses and volume of information lost}

In this section we determine the distribution of the total loss duration $\tau(x)$ in a busy period and the distribution of the total volume of information lost $V(x)$ in a busy period. From Section 2.2, we have

$$
\tau(x)=\sum_{k=1}^{K} \tau_{k}(x), \quad V(x)=\sum_{k=1}^{K} V_{k}(x) .
$$

To obtain the distribution of $\tau(x)$ and $V(x)$, we need some results about absorbing Markovian arrival processes. 


\subsection{The absorbing Markovian arrival process}

The Markovian arrival process is well known in the queueing literature and was introduced in [9]. Here, we consider a variant of this process in which we add an absorbing state. This process is defined as a continuous-time Markov chain $\{(N(t), J(t)), t \geq 0\}$ with $N(0)=0$ on the state space $S=\{(a, 0)\} \cup(\mathbb{N} \times\{1, \ldots, m\})$ with $m \geq 1$ and where state $(a, 0)$ is absorbing. Its infinitesimal generator $\boldsymbol{G}$ is given by

$$
\boldsymbol{G}=\left(\begin{array}{cccccccc}
0 & 0 & 0 & 0 & 0 & 0 & 0 & \ldots \\
\boldsymbol{V} & \boldsymbol{D}_{0} & \boldsymbol{D}_{1} & 0 & 0 & 0 & 0 & \ldots \\
\boldsymbol{V} & 0 & \boldsymbol{D}_{0} & \boldsymbol{D}_{1} & 0 & 0 & 0 & \ldots \\
\boldsymbol{V} & 0 & 0 & \boldsymbol{D}_{0} & \boldsymbol{D}_{1} & 0 & 0 & \ldots \\
\vdots & \vdots & \vdots & \vdots & \vdots & \ddots & \ddots & \ddots
\end{array}\right),
$$

where the first row and the first column correspond to the absorbing state $a$. Matrices $\boldsymbol{D}_{0}$ and $\boldsymbol{D}_{1}$ are nonzero $(m, m)$ matrices and column vector $\boldsymbol{V}$ is a nonzero vector with dimension $m$. The matrix $\boldsymbol{G}$ being an infinitesimal generator, we have $\boldsymbol{D}_{0} 1+\boldsymbol{D}_{1} 1+\boldsymbol{V}=0$. Moreover, we assume that all the states, except state $(a, 0)$, are transient. This implies in particular that matrix $\boldsymbol{D}_{0}$ is invertible. For $\ell \geq 0$, we define the subset of states $S_{\ell}=\{(\ell, 1), \ldots,(\ell, m)\}$. As in [13], we set $\xi_{0}=0$ and we denote, for all $n \geq 1$, by $\xi_{n}$ the instant at which either the $n$th arrival occurs or the absorbing state is reached. Variable $\xi_{n}$ is then defined by $\xi_{n}=\inf \{t \geq 0 \mid N(t) \in\{n, a\}\}$ for all $n \geq 0$. The $n$th interarrival time $\Gamma_{n}$ is then defined by $\Gamma_{n}=\xi_{n}-\xi_{n-1}$ for $n \geq 1$. We also define $Y$ as the number of subsets $S_{\ell}$ visited by the process until absorption, i.e.

$$
Y=\sum_{\ell=1}^{\infty} \mathbf{1}_{\left\{\Gamma_{\ell}>0\right\}}
$$

We then have $\xi_{n}=\xi_{Y}$ for $n \geq Y$ and, thus, $\Gamma_{Y}>0$ and $\Gamma_{n}=0$ for $n \geq Y+1$. Since $N(0)=0$, the initial probability distribution is concentrated on the states $(0,1), \ldots,(0, m)$. We denote by $\boldsymbol{\beta}$ the row vector of dimension $m$ defined by $\beta_{i}=\mathbb{P}\{J(0)=i\}$ for $i=1, \ldots, m$. Thus, we have $\beta_{1}+\cdots+\beta_{m}=1$. Following the results of [13], the joint distribution of $\Gamma_{1}, \ldots, \Gamma_{k}$ is given by

$$
\mathbb{P}\left\{\Gamma_{1}>t_{1}, \ldots, \Gamma_{k}>t_{k}\right\}=\boldsymbol{\beta}\left[\prod_{\ell=1}^{k-1} \mathrm{e}^{\boldsymbol{D}_{0} t_{\ell}}\left(-\boldsymbol{D}_{0}\right)^{-1} \boldsymbol{D}_{1}\right] \mathrm{e}^{\boldsymbol{D}_{0} t_{k}} \mathbb{1}
$$

We then have, for every $k \geq 0, \Gamma_{k+1}>0 \Longleftrightarrow Y>k$. By taking $t_{1}=\cdots=t_{k-1}=0$ and $t_{k}=t$ in (21), for every $k \geq 1$ and $t \geq 0$, we obtain

$$
\mathbb{P}\left\{\Gamma_{k}>t\right\}=\boldsymbol{\beta}\left(\left(-\boldsymbol{D}_{0}\right)^{-1} \boldsymbol{D}_{1}\right)^{k-1} \mathrm{e}^{\boldsymbol{D}_{0} t} \mathbb{1},
$$

i.e.

$$
\mathbb{P}\{Y>k\}=\boldsymbol{\beta}\left(\left(-\boldsymbol{D}_{0}\right)^{-1} \boldsymbol{D}_{1}\right)^{k} 1 .
$$

We denote by $\Gamma$ the total time spent by the process in the transient states, i.e. $\Gamma=\inf \{t \geq$ $0 \mid N(t)=a\}$. By the definition of $Y$, we have

$$
\Gamma=\sum_{k=1}^{Y} \Gamma_{k}=\sum_{k=1}^{\infty} \Gamma_{k}
$$


The distribution of $\Gamma$ is given, for every $t \geq 0$, by $\mathbb{P}\{\Gamma>t\}=(\boldsymbol{\beta}, 0,0, \ldots) \mathrm{e}^{\boldsymbol{L} t} \mathbb{1}$, where

$$
\boldsymbol{L}=\left(\begin{array}{ccccccc}
\boldsymbol{D}_{0} & \boldsymbol{D}_{1} & 0 & 0 & 0 & 0 & \ldots \\
0 & \boldsymbol{D}_{0} & \boldsymbol{D}_{1} & 0 & 0 & 0 & \ldots \\
0 & 0 & \boldsymbol{D}_{0} & \boldsymbol{D}_{1} & 0 & 0 & \ldots \\
\vdots & \vdots & \vdots & \vdots & \ddots & \ddots & \ddots
\end{array}\right)
$$

It is easily checked that for every $k \geq 0$, we have

$$
\boldsymbol{L}^{k} 1=\left(\begin{array}{c}
\left(\boldsymbol{D}_{0}+\boldsymbol{D}_{1}\right)^{k} \| \\
\left(\boldsymbol{D}_{0}+\boldsymbol{D}_{1}\right)^{k} \| \\
\vdots
\end{array}\right)
$$

We then obtain

$$
\mathbb{P}\{\Gamma>t\}=\boldsymbol{\beta} \mathrm{e}^{\left(\boldsymbol{D}_{0}+\boldsymbol{D}_{1}\right) t} 1 .
$$

\subsection{Distribution of $\tau(x)$ and $V(x)$}

Using the notation $\boldsymbol{a}(x)$ and $\boldsymbol{R}(x)$ introduced in (16), we obtain the following result.

Theorem 7. For every $v, t \geq 0$, we have

$$
\begin{gathered}
\mathbb{P}\{V(x)>v\}=\boldsymbol{\pi}^{+} \boldsymbol{M}(x) \mathrm{e}^{\left(\boldsymbol{Q}_{++}+\boldsymbol{Q}_{+-} \boldsymbol{H}(x)\right) v} 1, \\
\mathbb{P}\{\tau(x)>t\}=\boldsymbol{a}(x) \mathrm{e}^{(\boldsymbol{A + \boldsymbol { B } R}(x)) t} 1 .
\end{gathered}
$$

Proof. The joint distribution of the $V_{k}$, which is given by (14) is equal to the joint distribution of the $\Gamma_{k}$ given by (21) by taking $\boldsymbol{\beta}=\boldsymbol{\pi}^{+} \boldsymbol{M}(x), \boldsymbol{D}_{0}=\boldsymbol{Q}_{++}$, and $\boldsymbol{D}_{1}=\boldsymbol{Q}_{+-} \boldsymbol{H}(x)$. With these values, the distribution of $K$ given by (19) and the distribution of $Y$ given by (22) are identical. Thus, according to (23),

$$
\mathbb{P}\{V(x)>v\}=\boldsymbol{\pi}^{+} \boldsymbol{M}(x) \mathrm{e}^{\left(\boldsymbol{Q}_{++}+\boldsymbol{Q}_{+-} \boldsymbol{H}(x)\right) v} 1 .
$$

The joint distribution of the $\tau_{k}$ given by (16) is equal to the joint distribution of the $\Gamma_{k}$ given by (21) by taking $\boldsymbol{\beta}=\boldsymbol{a}(x), \boldsymbol{D}_{0}=\boldsymbol{A}$ and $\boldsymbol{D}_{1}=\boldsymbol{B} \boldsymbol{R}(x)$. With these values, the distribution of $K$ given by (20) and the distribution of $Y$ given by (22) are identical. Thus, according to (23), $\mathbb{P}\{\tau(x)>t\}=\boldsymbol{a}(x) \mathrm{e}^{(\boldsymbol{A}+\boldsymbol{B} \boldsymbol{R}(x)) t} 1$.

\subsection{Loss probability}

Let $\mathbb{E}_{\mathrm{IP}}$ and $\mathbb{E}_{\mathrm{BP}}$ be the mean durations of an idle period and a busy period, respectively, in the stationary regime and let $p$ be the stationary distribution of Markov chain $(\varphi(t))$. The mean input rate is then $\mathbb{E}(\chi)=\sum_{i \in S} p_{i} \chi_{i}$, where $\chi_{i}$ is the input rate in the queue when the modulating Markov chain $(\varphi(t))$ is in state $i$. The quantity $\pi_{\text {loss }}$, defined by (1), is then given by

$$
\pi_{\text {loss }}=\left(\frac{1}{\mathbb{E}_{\mathrm{IP}}+\mathbb{E}_{\mathrm{BP}}}\right)\left(\frac{\mathbb{E}(V(x))}{\mathbb{E}(\chi)}\right) .
$$

From (24), we have

$$
\mathbb{E}(V(x))=\boldsymbol{\pi}^{+} \boldsymbol{M}(x)\left(-\boldsymbol{Q}_{++}-\boldsymbol{Q}_{+-} \boldsymbol{H}(x)\right)^{-1} \mathbb{1} .
$$


Using (10) and (13), the mean duration of an idle period is given by

$$
\mathbb{E}_{\mathrm{IP}}=\left(\boldsymbol{\pi}^{-}, \boldsymbol{0}\right)\left(-\boldsymbol{A}^{\prime}\right)^{-1} \mathbb{\|}=\boldsymbol{\pi}^{-}\left(-\boldsymbol{Q}_{--}\right)^{-1} \boldsymbol{R}_{-}^{-1}\left(\boldsymbol{I}+\boldsymbol{T}_{-0}\left(-\boldsymbol{T}_{00}\right)^{-1}\right) \mathbb{1} .
$$

The mean durations $\mathbb{E}_{\mathrm{BP}}$ and $\mathbb{E}_{\mathrm{IP}}$ are related by the relation

$$
\mathbb{P}\{X=0\}=\frac{\mathbb{E}_{\mathrm{IP}}}{\mathbb{E}_{\mathrm{IP}}+\mathbb{E}_{\mathrm{BP}}},
$$

where $X$ is the fluid level in stationary regime. From (25), we have

$$
\pi_{\text {loss }}=\frac{\mathbb{P}\{X=0\} \mathbb{E}(V(x))}{\mathbb{E}_{\mathrm{IP}} \mathbb{E}(\chi)} .
$$

The distribution of $X$ and, more precisely, the quantity $\mathbb{P}\{X=0\}$ was obtained in [7] using matrix analytic methods.

To conclude this section, we note that the matrices $\boldsymbol{\Psi}(x), \boldsymbol{M}(x), \boldsymbol{H}(x)$, and $\boldsymbol{\Gamma}(x)$ are equivalent to the matrices $\boldsymbol{G}(0, x), \boldsymbol{H}(0, x)$, and $\boldsymbol{H}(x, x)$ in [4] and the matrix $\boldsymbol{\Psi}_{0}$ in [6], respectively. They can be evaluated efficiently using the explicit expressions in [2, Corollary 2] and [6, Theorem 3] (substitute $s=0$ ) and the quadratic algorithms in [3] and [5], and without the use of numerical inversion of the Laplace-Stieltjes transforms.

Matrices $\boldsymbol{\Psi}(x), \boldsymbol{H}(x)$, and $\boldsymbol{\Gamma}(x)$ are expressed here in terms of matrix differential Riccati equations that can be solved using the method described in [14] which consists of computing the exponential of the matrix $\boldsymbol{C} \boldsymbol{Q} x$ and then in solving a linear system in which the parameters are blocks of the matrix $\exp (\boldsymbol{C} \boldsymbol{Q} x)$. Matrix $\boldsymbol{M}(x)$ (see (7)), is the solution to a system of ordinary differential equations with nonconstant coefficient matrix that can easily be solved numerically using a classical Runge-Kutta fourth-order method available in MATLAB ${ }^{\circledR}$, for example.

\section{Conclusion}

In this paper we have developed a method of computing the distributions of the total duration of congestion and the volume of lost information in a finite buffer fed with a Markov-modulated fluid input process in the stationary regime. This allows us to exactly compute the metric $\pi_{\text {loss }}$ defined by (1) and corresponding to the fraction of lost information. In addition, we consider the stationary regime and study the state of the system, when a busy period starts; this aspect is not investigated in [10]. Finally, one outstanding result of this paper is that we have shown that the total loss duration and the total volume of information lost in a busy period have phase-type distributions.

\section{References}

[1] Asmussen, S. (1995). Stationary distributions for fluid flow models with or without Brownian noise. Commun. Statist. Stoch. Models 11, 21-49.

[2] Bean, N. G. and O'Reilly, M. M. (2008). Performance measures of a multi-layer Markovian fluid model. Ann. Operat. Res. 160, 99-120.

[3] Bean, N. G., O'Reilly, M. M. and Taylor, P. G. (2005). Algorithms for return probabilities for stochastic fluid flows. Stoch. Models 21, 149-184.

[4] Bean, N. G., O'Reilly, M. M. and Taylor, P. G. (2005). Hitting probabilities and hitting times for stochastic fluid flows. Stoch. Process. Appl. 115, 1530-1556.

[5] Bean, N. G., O'Reilly, M. M. and Taylor, P. G. (2008). Algorithms for the Laplace-Stieltjes transforms of first return times for stochastic fluid flows. Method. Comput. Appl. Prob. 10, 381-408.

[6] Bean, N. G., O’Reilly, M. M. and Taylor, P. G. (2009). Hitting probabilities and hitting times for stochastic fluid flows: the bounded model. Prob. Eng. Inf. Sci. 23, 121-147. 
[7] Da Silva Soares, A. And Latouche, G. (2006). Matrix-analytic methods for fluid queues with finite buffers. Performance Evaluation 63, 295-314.

[8] Guillemin, F. and Sericola, B. (2012). On the fluid queue driven by an ergodic birth and death process. In Telecommunications Networks - Current Status and Future Trends, In Tech, Rijeka, pp. 379-404.

[9] Neuts, M. F. (1979). A versatile Markovian point process. J. Appl. Prob. 16, 764-779.

[10] O’Reilly, M. M. and Palmowski, Z. (2013). Loss rates for stochastic fluid models. Performance Evaluation 70, 593-606.

[11] Rogers, L. C. G. (1994). Fluid models in queueing theory and Wiener-Hopf factorization of Markov chains. Adv. Appl. Prob. 4, 390-413.

[12] Rubino, G. ANd Sericola, B. (1989). Accumulated reward over the $\mathrm{n}$ first operational periods in fault-tolerant computing systems. Res. Rep. 1028, INRIA.

[13] Sericola, B. (2013). Markov Chains: Theory, Algorithms and Applications. Iste-Wiley, London.

[14] Sericola, B. and Remiche, M.-A. (2011). Maximum level and hitting probabilities in stochastic fluid flows using matrix differential Riccati equations. Method. Comput. Appl. Prob. 13, 307-328. 